\title{
A cohort study of hepatectomy-related complications and prediction model for postoperative liver failure after major liver resection in 1,441 patients without obstructive jaundice
}

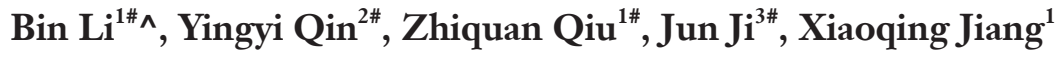 \\ ${ }^{1}$ Biliary Tract Surgery Department I, Eastern Hepatobiliary Surgery Hospital, Secondary Military Medical University, Shanghai, China; ${ }^{2}$ Department \\ of Health Statistics, Second Military Medical University, Shanghai, China; ${ }^{3}$ Laboratory diagnosis Department, Eastern Hepatobiliary Surgery \\ Hospital, Secondary Military Medical University, Shanghai, China \\ Contributions: (I) Conception and design: X Jiang, B Li; (II) Administrative support: X Jiang; (III) Provision of study materials or patients: B Li, \\ Z Qiu; (IV) Collection and assembly of data: Z Qiu, J Ji; (V) Data analysis and interpretation: Y Qin, B Li; (VI) Manuscript writing: All authors; (VII) \\ Final approval of manuscript: All authors. \\ \#These authors contributed equally to this work. \\ Correspondence to: Xiaoqing Jiang; Bin Li. Biliary Tract Surgery Department I, Eastern Hepatobiliary Surgery Hospital, Secondary Military Medical \\ University, No. 225 Changhai Road, Shanghai 200438, China. Email: jxq1225@sina.com; libinjeff@126.com.
}

Background: This cohort study, based on a large sample of extensive hepatectomy cases, aimed to analyze the distribution of hepatectomy-related complications and to develop a predictive model of posthepatectomy liver failure (PHLF).

Methods: Data of patients who underwent hepatectomy of $\geq 3$ liver segments at the Eastern Hepatobiliary Surgery Hospital from 2000 to 2016 were collected and analyzed. Information on hepatectomy-related complications was collected and risk factors were analyzed. A total of 1,441 eligible patients were randomly assigned at 3:1 ratio into the derivation $(n=1,080)$ and validation $(n=361)$ cohorts. The multivariable logistic regression model was used to establish the prediction model of PHLF in the derivation cohort.

Results: The incidence rates of PHLF, ascites, bile leakage, intra-abdominal bleeding, and abscesses were $58.22 \%, 10.76 \%, 11.17 \%, 9.71 \%$, and $4.16 \%$, respectively. The 90 -day perioperative mortality rate was $1.32 \%$. Multivariate analyses found that age, gender, platelet, creatinine, gamma-glutamyltransferase, thrombin time, fibrinogen, hepatitis B e (HBe) antigen positive, and number of resected liver segments were independent prognostic factors of PHLF in the derivation cohort and included in the nomogram. The prediction model demonstrated good discrimination [area under the curve $=0.726,95 \%$ confidence interval (CI), 0.696-0.760, $\mathrm{P}<0.0001]$ and calibration.

Conclusions: Our study showed a high perioperative safety and a low risk of serious complications in patients who underwent major liver resection (MLR) at a large hepatobiliary surgery center. Routine preoperative clinical information can be used to develop a postoperative liver failure risk prediction model for rational planning of surgery.

Keywords: Major liver resection (MLR); postoperative complications; liver failure; predictive model; nomogram

Submitted Jul 23, 2020. Accepted for publication Nov 20, 2020.

doi: $10.21037 / \mathrm{atm}-20-5472$

View this article at: http://dx.doi.org/10.21037/atm-20-5472

^ ORCID: 0000-0002-7415-629X. 


\section{Introduction}

Hepatectomy is a curative treatment of benign and malignant primary hepatobiliary tumors, metastatic liver tumors, intrahepatic bile duct stones, and other hepatobiliary system diseases. Complications such as posthepatectomy liver failure (PHLF), posthepatectomy hemorrhage $(\mathrm{PHH})$, and postoperative death are serious adverse medical events after hepatectomy. Therefore, there is a need to identify the risk factors associated with serious complications, such as PHLF, to effectively identify highrisk patients and reduce the risk of postoperative adverse complications (1).

However, the use of different PHLF criteria, different perioperative time cut-offs for death, and different hepatic resection ranges will result in clinical heterogeneity between studies. This is also the main reason for the large data span for the incidence of PHLF and perioperative mortality in previous literature (i.e., from $0 \%$ to $43.1 \%$ for the former and from $0.5 \%$ to $15.6 \%$ for the latter) (2).

There is increasing awareness of the importance and value of generating uniform definitions of outcome parameters to enable reliable comparison of the results from different studies and ultimately to provide patients with the best available therapy. Postoperative ascites, liver failure, bile leakage, intra-abdominal bleeding, intraabdominal abscesses, and perioperative death were major adverse events after hepatectomy. The inclusion of these complications as a primary study component not only allows for a controlled analysis of the results of relevant studies, but also contributes to the standardization of study design and comparability of results in randomized controlled trials of liver surgery (3). In 2011, the International Research Group of Liver Surgery (ISGLS) developed definitions and classification criteria for PHH (4), posthepatectomy biliary leakage (PHBL) (5), and PHLF (6), which were supported by other findings (7). The above work laid a foundation for the standardized assessment of hepatectomy-related risk factors and clinical outcomes.

Although expanding the scope of hepatectomy improves the radical resection rate of the liver lesion site, it will inevitably lead to an increased risk of postoperative complications due to reduced residual liver function. Therefore, a comprehensive and accurate assessment of the risk factors and clinical outcomes of major liver resection (MLR) is very important for decision-making on the necessity of liver surgery and the formulation of clinical intervention pathways for hepatectomy-related complications.

Previous studies have constructed predictive models of PHLF for specific hepatectomy populations such as primary hepatocellular carcinoma (8), patients with cirrhosis (9), specific laboratory test values, and/or imaging features (10).

In this cohort study, we used a large sample of extensive hepatectomy cases to analyze the distribution of hepatectomy-related complications and to develop a predictive model of PHLF and to validate the predictive efficacy of the model. We present the following article in accordance with the TRIPOD reporting checklist (available at http://dx.doi.org/10.21037/atm-20-5472).

\section{Methods}

\section{Source of data}

Data were retrieved from electronic databases from 2000 to 2016 using the medical records archives and database of the department of laboratory diagnostics of the Eastern Hepatobiliary Surgery Hospital. Information on patients who underwent MLR at the Eastern Hepatobiliary Surgery Hospital of the Second Military Medical University during the study period and the data on each patient combined with the information recorded in the original medical records were verified. Data from 1,441 patients eligible for the study were eventually included in this cohort study. This study conformed to the provisions of the Declaration of Helsinki (as revised in 2013). This study has been approved by the Ethics Committee of Eastern Hepatobiliary Surgery Hospital (Ethics Audit No. EHBHKY2020-K-004). Each patient signed the clinical study informed consent form in person or by proxy.

\section{Participants}

All cases undergoing hepatectomy included in this study were classified into the following six categories according to their pathological diagnosis: hepatocellular carcinoma, intrahepatic cholangiocarcinoma, hepatic hemangioma, intrahepatic cholelithiasis, metastatic hepatoma, and other rare occupational diseases of the hepatobiliary system.

The inclusion criteria were as follows: (I) age $>18$ years, (II) hepatectomy of at least three liver segments, (III) available perioperative follow-up data, (IV) absence of significant anemia, infection, and renal insufficiency decompensation (serum creatinine level $\leq 177 \mu \mathrm{mol} / \mathrm{L}$ ) before hepatectomy, and (V) Child-Pugh grades A and B for liver function. The 
exclusion criteria were as follows: (I) presence of obstructive jaundice, (II) previous portal vein embolization or ligation before hepatectomy, and (III) history of associating liver partition and portal vein ligation for staged hepatectomy.

\section{Outcome}

The objective of this study was to construct a model with a predictive power exceeding 0.7 for PHLF which can also effectively distinguish between PHLF grades A, B, and C.

\section{Predictors}

Data included demographic and preoperative, intraoperative, and postoperative information. Demographic information included age, gender, and blood type (Table S1). Preoperative information included (I) patient's body mass index (BMI) value, presence of diabetes, history of chronic heavy alcohol consumption, history of chemotherapy, presence of cirrhosis, and presence of combined ascites, etc., and (II) laboratory test results for blood routine, liver function, kidney function, electrolytes, etc. (Table S1). Potentially relevant intraoperative risk factors included the number of hepatic segments resected, whether or not to perform hepatic blood flow blockade by Pringle maneuver, whether or not to perform intermittent Pringle maneuver, total Pringle time, intraoperative bleeding volume, and intraoperative blood transfusion volume (Table S2).

Postoperative information included hepatectomy-related complications and perioperative mortality (Table S2). This study categorized ascites, bile leakage, intra-abdominal hemorrhage, abscesses, liver failure, and death 90 days after hepatectomy as major complications according to previous studies (3).

According to the definition of ISGLS, the occurrence of complications such as liver failure (ISGLS-PHLF) (6), bile leakage (ISGLS-PHBL) (5), and hemorrhage (ISGLS$\mathrm{PHH})$ was determined and were further classified into grades A, B, and C (4).

According to Couinaud segmentation classification (11), the liver lobe is divided into 1-8 segments. For patients who underwent complete liver segmental resection combined with local resection of tumor subsites, segmental wedge resection, and other liver resections of areas that did not reach the whole liver segment, they were uniformly described as liver segmental resection $3+, 4+, 5+, 6+$, etc. Extended left hemihepatectomy, right hemihepatectomy, and simultaneous wedge resection or enucleation of subfocals in other lobes were equivalent to extended segmentectomy and labeled (+).

The laboratory index was treated as a continuous variable when values in most of the patients were in the normal range $(\geq 85 \%)$. For the remaining indices, the restricted cubic spline analyses with three knots $\left(25^{\text {th }}\right.$, $50^{\text {th }}, 75^{\text {th }}$ percentiles) were applied to detect the nonlinear relationship between laboratory index and ISGLS-PHLF. The laboratory index was also treated as a continuous variable for those without nonlinear relationship. For the laboratory index with significant nonlinear relationship, the cut-off value was established according to the following steps: non-PHLF patients of the total cohort were divided equally into 10 parts to obtain nine cut-off values based on the index, the prevalence of PHLF for each part was calculated, and cut-off categories with similar prevalence rates were combined. The final cut-off value was determined based on the normal range and categories combined: preoperative serum hemoglobin level $(<130$, $\geq 130 \mathrm{~g} / \mathrm{L})$, total bile acid $(<2.7,2.7-5.4, \geq 5.4 \mu \mathrm{mol} / \mathrm{mL})$, alanine aminotransferase $(<50, \geq 50 \mathrm{U} / \mathrm{L})$, aspartic acid transferase $(<40, \geq 40 \mathrm{U} / \mathrm{L})$, lactate dehydrogenase $(<250$, $\geq 250 \mathrm{U} / \mathrm{L})$, and gamma-glutamyltransferase (GGT) $(<60$, $60-180, \geq 180 \mathrm{U} / \mathrm{L})$.

\section{Sample size}

In this study, we included 1,578 patients who underwent MLR at the Eastern Hepatobiliary Surgery Hospital during the study cycle for whom complete perioperative followup data were available. The total cohort was randomly divided based on a 3:1 ratio into the derivation cohort and validation cohort (Figure 1).

\section{Missing data}

The purpose of this study was to analyze the occurrence of complications associated with MLR and to develop a predictive model of liver failure after hepatectomy using routine preoperative clinical information from patients. Therefore, according to the inclusion criteria of this cohort study, 137 cases that failed to obtain all baseline characteristics related data needed for the study were excluded from this study.

\section{Statistical analysis methods}

The median (quartile) for continuous variables and 


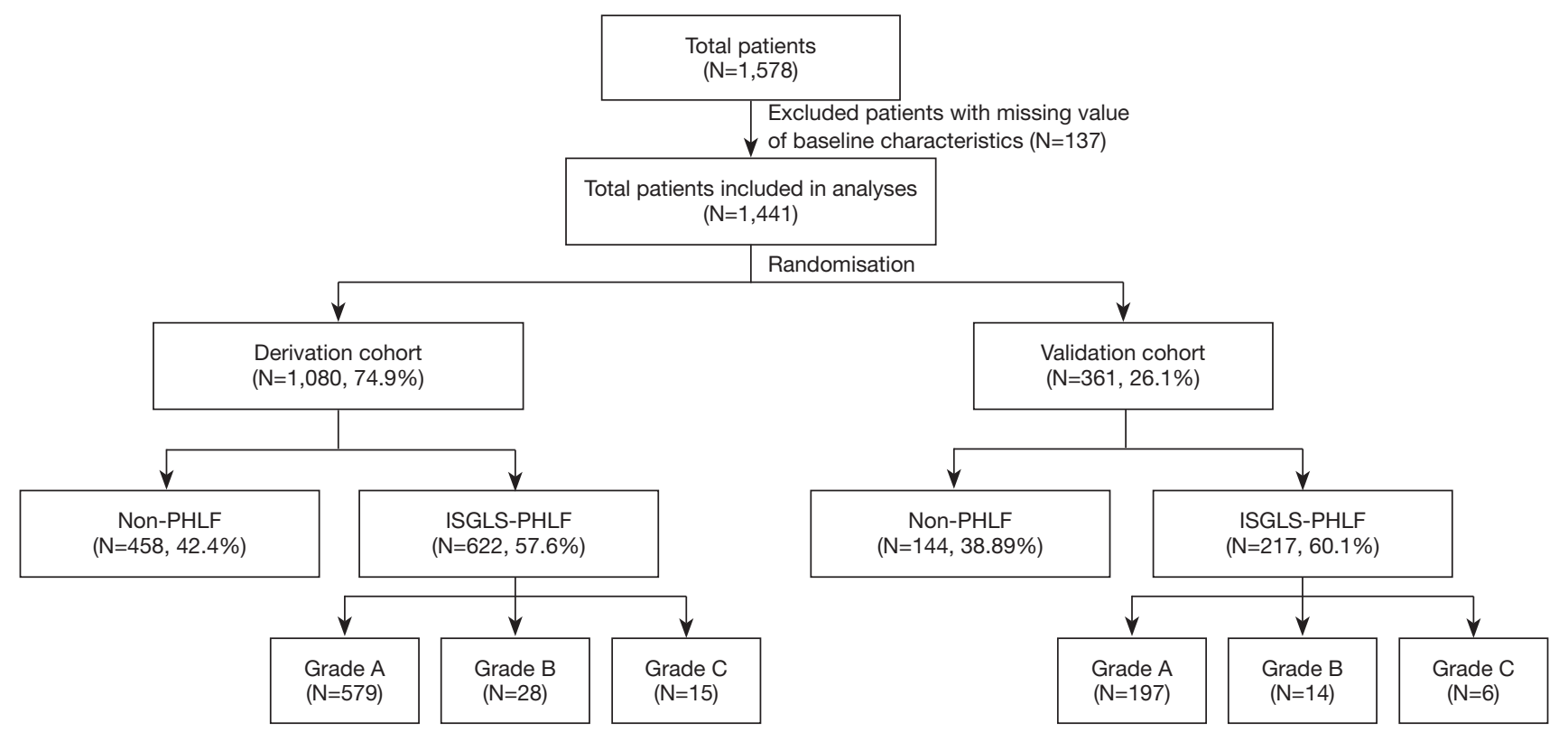

Figure 1 Flow chart of 1,441 patients included in the analyses. PHLF, posthepatectomy liver failure; ISGLS, International Research Group of Liver Surgery.

frequencies and percentages for categorical variables were calculated. The Wilcoxon rank-sum test, chi-square test, or Cochran-Mantel-Haenszel test were used to compare demographics and baseline characteristics between the derivation cohort and validation cohort.

The prediction model of ISGLS-PHLF was established based on the derivation cohort. Candidate variables were all preoperative factors and demographic information. Univariate analyses were performed to select the potential risk factors of PHLF $(\mathrm{P}<0.2)$. All potential risk factors were included in the binary logistic backward stepwise regression model $\left(\alpha_{s l s}=0.05\right)$ to obtain an optimal prediction model. The evaluation for the performance of the prediction model was based on scaled Brier score, $R^{2}$, slope, and intercept in the derivation cohort and validation cohort with 1,000-bootstrap resampling method. Calibration was evaluated by the HosmerLemeshow goodness-of-fit test and calibration plots in both derivation and validation cohorts (12). The nomogram was established for the prediction model, and the total points for each patient was calculated. Receiver operating characteristic (ROC) analyses were performed to evaluate the performance of the prediction model in both the derivation and validation cohorts. ROC analyses were also performed for different ISGLS-PHLF grades and patient subgroups (tumor and non-tumor disease) in the total cohort. Additionally, multivariable logistic regression model was used to detect the risk factors of posthepatectomy outcomes.

The nomogram, ROC analyses, and evaluation of the prediction model were performed using $\mathrm{R}$ version 3.5.2 ( $\mathrm{R}$ Foundation for Statistical Computing, Vienna, Austria). The remaining statistical analyses were performed using SAS software version 9.4 (SAS Institute Inc., Cary, NC). All reported $\mathrm{P}$ values were two-sided, and $\mathrm{P}<0.05$ was regarded as statistically significant.

\section{Risk groups}

Patients who undergo hepatectomy are at risk of postoperative liver failure. Clinical practice and research have found that the risk of postoperative liver failure increases with the expansion of the scope of hepatectomy. In this study, we performed multivariable analyses of risk factors associated with postoperative liver failure in a population of patients with resected liver parenchyma $\geq 3$ complete liver segments, and a postoperative liver failure prediction model was developed and validated. Therefore, in this study, both the derivation cohort and validation cohort were risk groups. 


\section{Development vs. validation}

All samples in this cohort study were from the same research institution, and there was a high degree of homogeneity in terms of clinical test quality control criteria, surgical protocols, and postoperative management criteria. As a result, data from the development cohort and validation cohort did not differ significantly in terms of setting, eligibility criteria, outcomes, or predictors.

\section{Results}

\section{Participants}

A total of 1,578 patients underwent extensive hepatectomy and perioperative follow-up information was obtained, excluding 137 patients with missing data, resulting in 1,441 patients being enrolled in the study.

A total of 1,441 MLR cases treated at the Eastern Hepatobiliary Surgery Hospital of the Second Military Medical University between 2000 and 2016 were screened as eligible cases for enrollment in this study. There were 939 male patients $(65.2 \%)$ and 502 female patients (34.8\%). The median age of all patients was 52 years. The disease types in 1,441 consecutive patients were distributed as follows: $50.52 \%(n=728)$ of the patients had hepatocellular carcinoma, $19.36 \%(\mathrm{n}=279)$ had intrahepatic cholangiocarcinoma, $18.74 \%(\mathrm{n}=270)$ had intrahepatic cholangiolithiasis, $4.09 \%(\mathrm{n}=59)$ had hepatic hemangioma, $2.22 \%(\mathrm{n}=32)$ had metastatic hepatoma, and $5.07 \%(\mathrm{n}=73)$ had other diseases of the hepatobiliary system. Common bile duct resection, choledochojejunostomy, regional lymph node dissection, colorectal resection, and diaphragm resection were the most common simultaneous operations. Demographic and clinicopathologic data are shown in Table S1.

Hepatectomy-related complications are shown in Table S2. The incidence rates of ISGLS-PHLF, ISGLSPHH, ISGLS-PHBL, postoperative ascites, and intraabdominal abscess were $58.22 \%, 10.76 \%, 11.17 \%, 9.71 \%$, and $4.16 \%$, respectively. A total of 80 patients $(5.55 \%)$ underwent postoperative thoracentesis drainage, including 79 patients who underwent right thoracentesis drainage and one left thoracentesis drainage. The 90 -day perioperative mortality rate was $1.32 \%$.

Among ISGLS-PHLF patients, the incidence of grade A PHLF requiring no change in the clinical management was $53.85 \%$, and the incidence of grade B PHLF resulting in a deviation from the regular clinical management but manageable without invasive treatment was $2.91 \%$. The incidence of grade C PHLF, which caused serious adverse effects on postoperative recovery, was $1.46 \%$. Similarly, the incidence of grade $\mathrm{C}$ defined as requiring intervention (e.g., embolization) or re-laparotomy was $0.69 \%$ in patients who developed ISGLS-PHH.

Most of the ISGLS-PHBL patients had grade B PHBL that required interventional treatment or the duration of bile leakage was more than 1 week, with an incidence rate of $9.51 \%$. The incidence rates of grade A PHBL that spontaneously healed within 1 week postoperatively and grade C PHBL requiring re-laparotomy were $1.53 \%$ and $0.14 \%$, respectively.

The multivariable analyses of posthepatectomy outcomes in the total cohort are shown in Table S3.

\section{Model development}

In the derivation cohort, 31 variables were potentially associated with ISGLS-PHLF based on the univariate analyses $(\mathrm{P}<0.2$, Table 1). After including all 31 variables in the binary logistic backward stepwise regression model, the optimal prediction model was established with nine variables: age, gender, preoperative serum laboratory test item (including platelet, creatinine, GGT, fibrinogen, thrombin time, hepatitis B e (HBe) antigen, and number of resected liver segments (Table 2).

\section{Model specification}

The nomogram was constructed based on the nine variables mentioned above that were strongly associated with ISGLSPHLF (Figure 2). The prediction model demonstrated good performance.

Each variable included in the nomogram has a unique corresponding line segment, and the total length of the segment reflects the contribution of that variable factor to ISGLS-PHLF. Each variable is marked with a scale score, and the individual scores for each variable in the sample at different values are summed to give a total score for the prediction, which indicates the probability of ISGLS-PHLF in the sample.

\section{Model performance}

The areas under the ROC curve (AUC) of the derivation and validation cohorts were 0.726 [95\% confidence interval (CI), 0.696-0.760] and 0.717 (95\% CI, 0.663-0.770), respectively. The calibration plots and Hosmer-Lemeshow 
Table 1 Univariate analyses of demographics and baseline characteristics in the derivation cohort

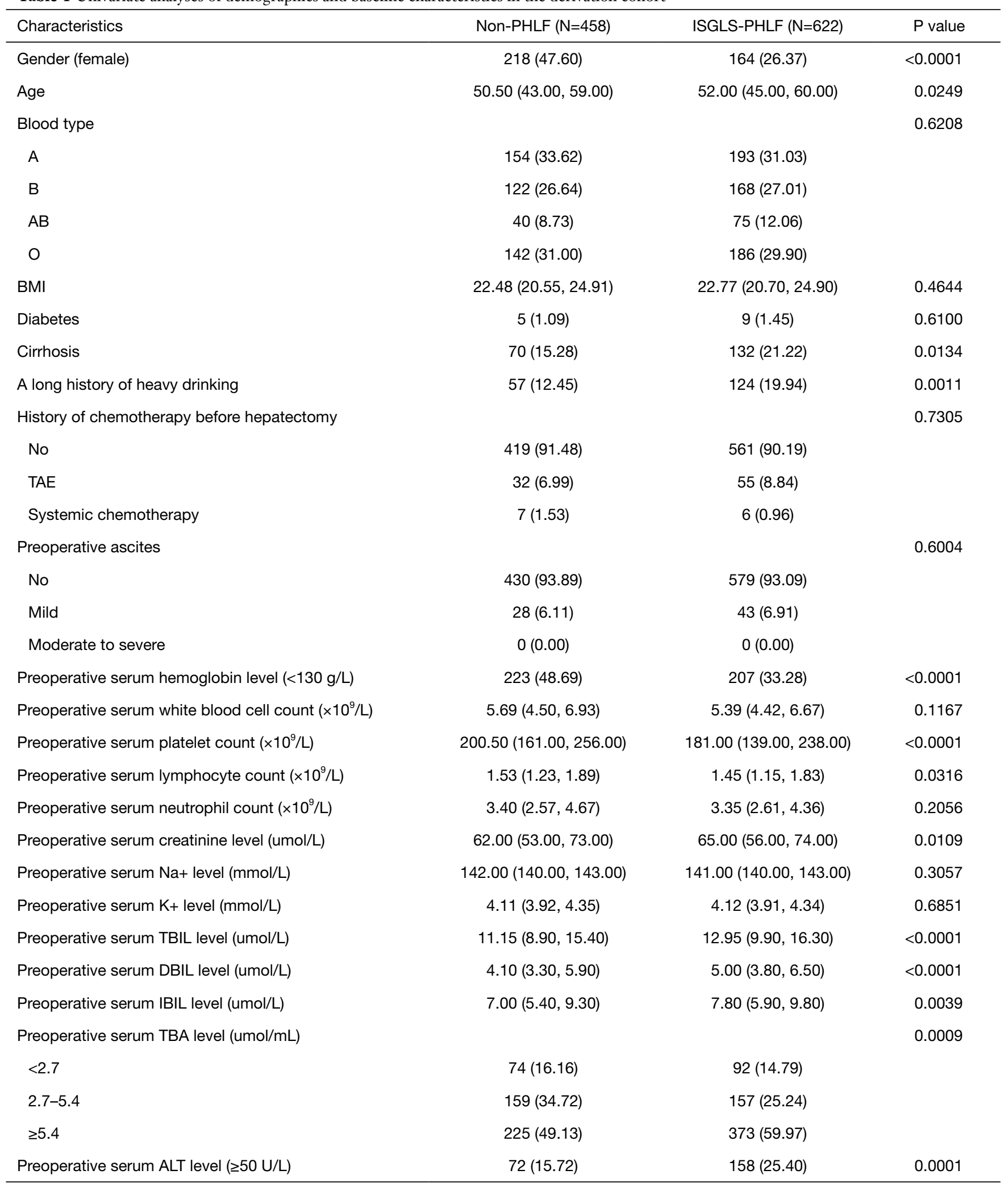

Table 1 (continued) 
Table 1 (continued)

\begin{tabular}{|c|c|c|c|}
\hline Characteristics & Non-PHLF (N=458) & ISGLS-PHLF (N=622) & $P$ value \\
\hline Preoperative serum LDH level ( $\geq 250$ U/L) & $66(14.41)$ & $98(15.76)$ & 0.5427 \\
\hline Preoperative serum GGT level (U/L) & & & $<0.0001$ \\
\hline$<60$ & $181(39.52)$ & $170(27.33)$ & \\
\hline$\geq 180$ & 90 (19.65) & $198(31.83)$ & \\
\hline Preoperative serum TP level (g/L) & $70.40(66.70,74.60)$ & $69.70(65.90,73.20)$ & 0.0175 \\
\hline Preoperative serum ALB level (g/L) & $42.00(39.20,44.30)$ & $41.25(38.90,43.60)$ & 0.0165 \\
\hline Preoperative serum GLB level (g/L) & $28.10(25.40,32.00)$ & $28.10(25.30,31.00)$ & 0.4414 \\
\hline APTT (s) & $26.70(24.60,29.70)$ & $27.50(25.10,30.30)$ & 0.0074 \\
\hline$\pi(\mathrm{s})$ & $19.00(18.10,19.90)$ & $19.30(18.40,20.20)$ & $<0.0001$ \\
\hline PT (s) & $11.40(10.80,12.20)$ & $11.50(11.00,12.30)$ & 0.0798 \\
\hline INR & $0.95(0.90,1.01)$ & $0.97(0.92,1.02)$ & 0.0461 \\
\hline Child-Pugh grade & & & 0.9385 \\
\hline Grade A & $454(99.13)$ & $618(99.36)$ & \\
\hline Grade B & $4(0.87)$ & $4(0.64)$ & \\
\hline HCV-Ab (positive) & $6(1.31)$ & $9(1.45)$ & 0.8493 \\
\hline Liver segment count excised & & & $<0.0001$ \\
\hline 3 & $288(62.88)$ & $261(41.96)$ & \\
\hline $3+$ & $45(9.83)$ & $50(8.04)$ & \\
\hline 4 & $99(21.62)$ & $219(35.21)$ & \\
\hline $4+$ & $18(3.93)$ & $64(10.29)$ & \\
\hline$\geq 5$ & $8(1.75)$ & $28(4.50)$ & \\
\hline $\begin{array}{l}\text { The segment of liver planned for resection has atrophied } \\
\text { (yes) }\end{array}$ & $124(27.07)$ & $124(19.94)$ & 0.0058 \\
\hline $\begin{array}{l}\text { The planned reserved segment of the liver has developed } \\
\text { compensatory hyperplasia (yes) }\end{array}$ & $21(4.59)$ & $32(5.14)$ & 0.6740 \\
\hline Pathological diagnostic classification & & & $<0.0001$ \\
\hline
\end{tabular}

Table 1 (continued) 
Table 1 (continued)

\begin{tabular}{lcc}
\hline Characteristics & Non-PHLF (N=458) & ISGLS-PHLF (N=622) \\
Hepatocellular carcinoma & $172(37.55)$ & $363(58.36)$ \\
Intrahepatic cholangiocarcinoma & $106(23.14)$ & $98(15.76)$ \\
Hepatic hemangioma & $24(5.24)$ & $24(3.86)$ \\
Intrahepatic cholelithiasis & $115(25.11)$ & $98(15.76)$ \\
Metastatic hepatoma & $11(2.40)$ & $12(1.93)$ \\
Other space-occupying diseases of the liver and biliary & $30(6.55)$ & $27(4.34)$ \\
\hline system &
\end{tabular}

Data are shown as $\mathrm{n}(\%)$ or median (range). $\mathrm{Na}+$, sodium; $\mathrm{K}+$, potassium; TBIL, total bilirubin; DBIL, direct bilirubin; IBIL, indirect bilirubin; TBA, total bile acid; ALT, alanine aminotransferase; AST, aspartic acid transferase; LDH, lactate dehydrogenase; GGT, gamma-glutamyltransferase; TP, total protein; ALB, albumin; GLB, globulin; pALB, prealbumin; APTT, activated partial thromboplastin; TT, thrombin time; $\mathrm{PT}$, prothrombin time; INR, international standardized ratio; HCV-Ab, hepatitis $\mathrm{C}$ antibody; HBs-Ag, hepatitis $\mathrm{B}$ surface antigen; HBs-Ab, hepatitis B surface antibody; HBe-Ag, hepatitis B e antigen; HBe-Ab, hepatitis B e antibody; HBc-Ab, hepatitis B core antibody.

goodness-of-fit test $\left(\chi^{2}=6.57, \mathrm{P}=0.5835\right)$ indicated that the prediction model was well calibrated. Details of model performance in the validation cohort and internal validation with resampling method are shown in Table 3, Figure S1, and Figure S2.

The total points of each patient in the total cohort was calculated based on the nomogram. The box plot showed significant difference between all pairwise cases except for grade $\mathrm{B} v s$. grade $\mathrm{C}$ cases (Figure $\mathrm{S} 3 \mathrm{~A})$. The ROC analysis for the prediction of each grade of PHLF indicated that patients with higher total points in the prediction model was likely to have higher grade of PHLF ( $\geq$ grade A, AUC 0.724, cut-off points $172.63 ; \geq$ grade B, AUC 0.792 , cut-off points 188.57 ; $\geq$ grade C, AUC 0.821, cut-off points 209.02; Table 4 and Figure S3B). Additionally, ROC analysis was conducted in subgroups of pathological diagnostic classification and revealed that the AUC in subgroup with tumor disease was similar to that of the total cohort (Figure S4).

\section{Risk factors associated with other bepatectomy-related complications}

Data from this group show that as the scope of hepatectomy increases, the number of risk factors for postoperative ascites, $\mathrm{PHBL}$, and $\mathrm{PHH}$ are higher, and the risk of postoperative massive pleural effusion increases. Patients with a history of preoperative chemotherapy, $>4$ resected liver segments, or resected liver lobes that have developed atrophy are at an increased risk of developing PHBL. This study also found a significantly higher risk of PHBL in patients without cirrhosis than in patients with cirrhosis, suggesting that cirrhosis was a protective factor in reducing PHBL in patients who underwent MLR.

The risk of postoperative intra-abdominal abscesses was higher in patients who developed atrophy of the liver lobe indicated for preoperative resection and/or patients who have developed compensatory hyperplasia of the liver lobe indicated for retention than in patients without these conditions (Table S3). High preoperative serum level of GGT is a risk factor of not only PHLF, but also postoperative intra-abdominal abscesses when the preoperative GGT value exceeds three times the upper limit of the normal value in laboratory test.

Patients with higher preoperative serum lymphocyte counts are also at increased risk for postoperative intraabdominal abscesses. An increased risk of PHH occurs when the preoperative thrombin time is significantly prolonged or the serum hemoglobin concentration is below 130 g/L. Patients with low preoperative serum prealbumin concentrations are more likely to develop massive pleural effusion and ascites after hepatectomy. In addition, patients with low preoperative serum sodium levels are prone to postoperative massive pleural effusion (Table S3).

\section{Discussion}

\section{Limitations}

Our study focused on a population with different backgrounds of hepatobiliary system disease, enrolled patients undergoing MLR as a study subject, clarified the 
Table 2 Final prediction model of posthepatectomy liver failure in the derivation cohort

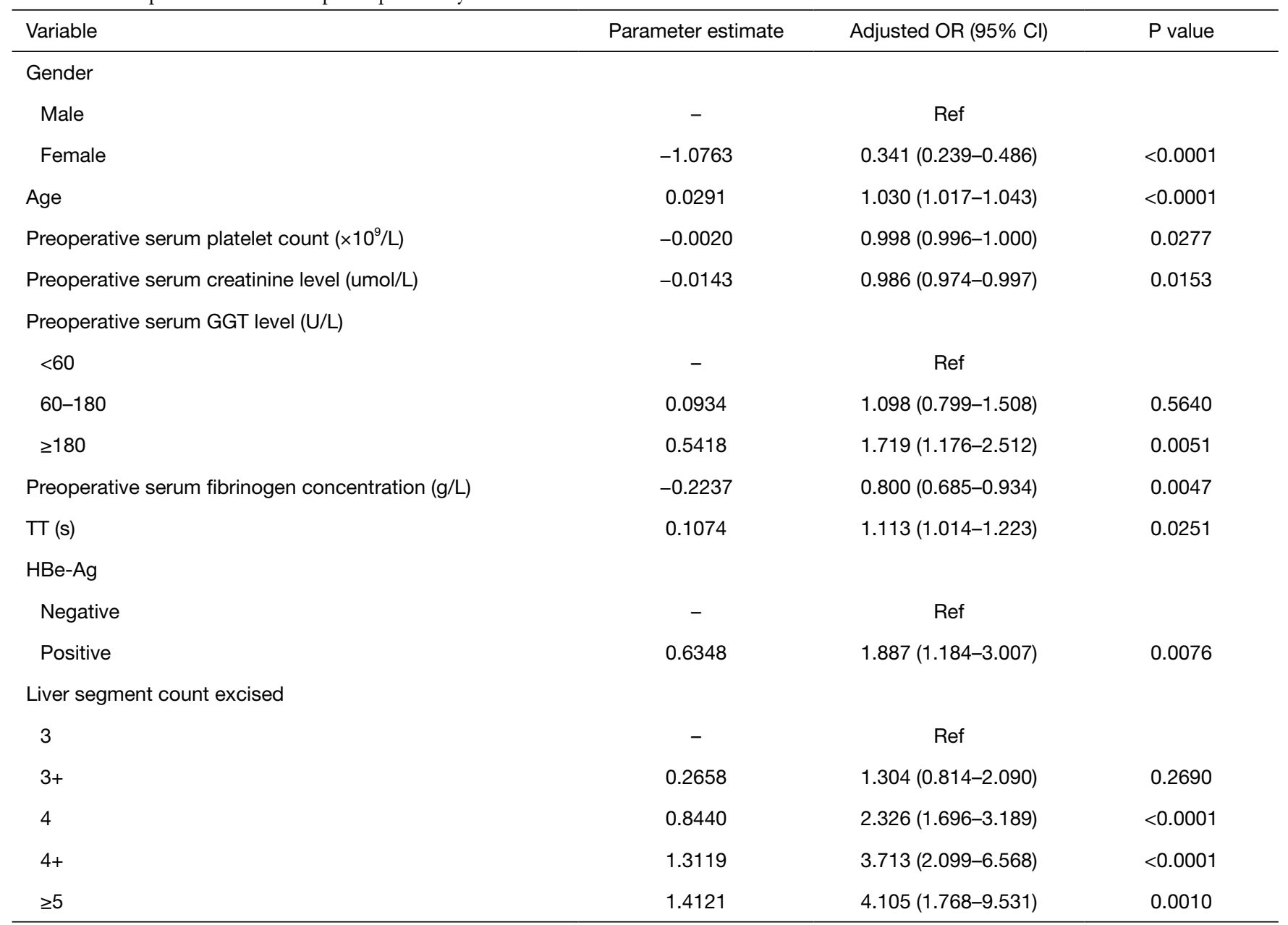

The criterion for variables in Table 1 included in multivariable logistic regression is: $\mathrm{P}$ value of univariate analysis $\leq 0.20$. The backward stepwise selection method was used in multivariable logistic regression ( $\alpha$ sls=0.05). OR, odds ratio; GGT, gamma-glutamyltransferase; $\Pi$, thrombin time; HBe-Ag, hepatitis B e antigen.

status of postoperative complications through a large cohort analysis, and attempted the feasibility of constructing a predictive model for PHLF using common information such as demographic information and clinically accessible preoperative laboratory test values.

This study has some limitations. First, the AUC value of the PHIL prediction model established in this study remains suboptimal $(<0.80)$. We also tried to increase the number of factors by including variables, such as intraoperative hemorrhage, whether the Pringle maneuver was performed and the total Pringle time, intraoperative hemorrhage, blood transfusion, etc., which may have significant effects on PHLF, in the analysis and established a prediction model of PHLF, but the results showed that the AUCs of the prediction model were also less than 0.8 . The above results indicate that other unclear risk factors are still affecting the occurrence of PHLF and included in the prediction model. Second, this study established a prediction model for PHLF based solely on the results of a single-center large cohort analysis. While the predictive efficacy of the model has been validated by an internal cohort, the validity and accuracy of the predictive model is subject to validation by an external cohort and further prospective studies.

\section{Interpretation}

The liver, as a key hub for many physiological processes, is one of the vital organs of the body. Liver-regulated 


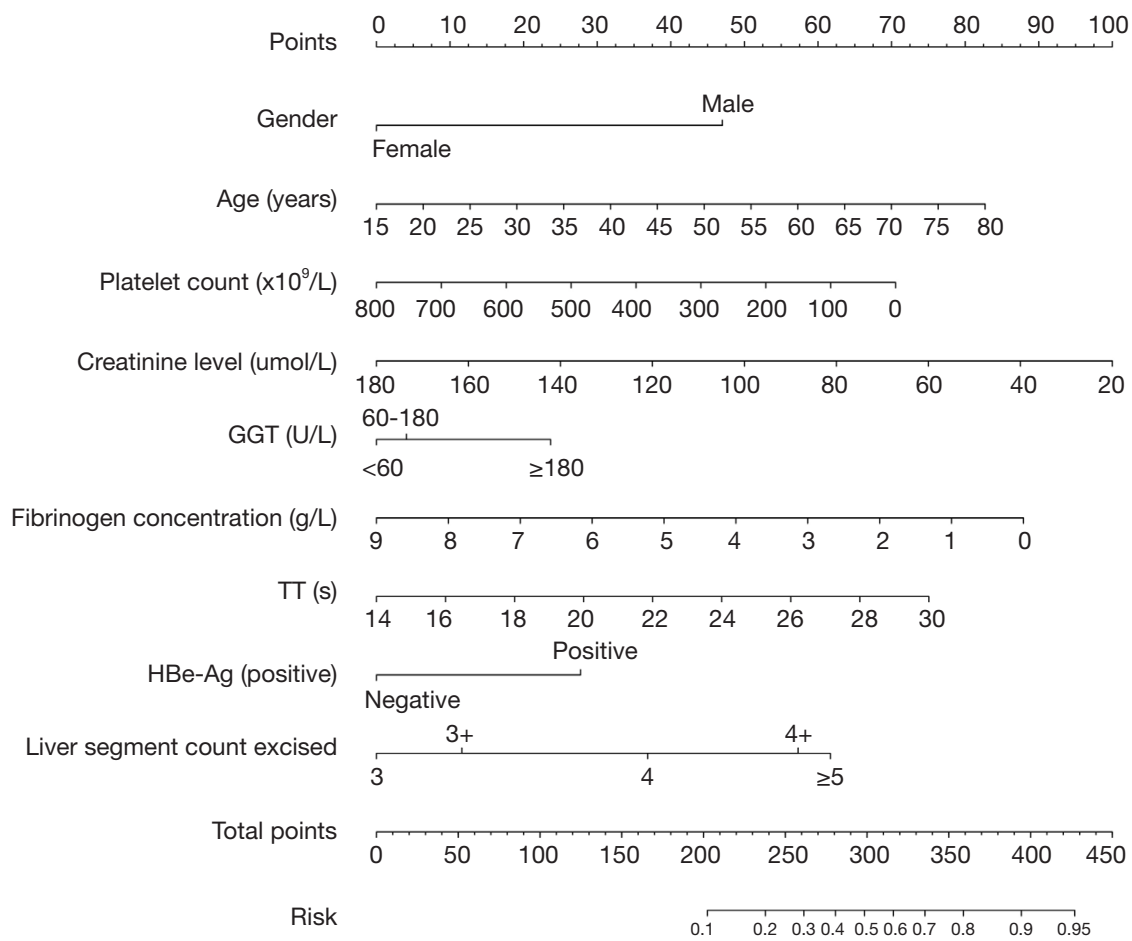

Figure 2 Nomogram for the prediction model of posthepatectomy liver failure. GGT, gamma-glutamyltransferase; TT, thrombin time.

Table 3 Performance for prediction model of posthepatectomy liver failure

\begin{tabular}{lcccc}
\hline \multirow{2}{*}{ Performance index } & Derivation cohort & Validation cohort & \multicolumn{2}{c}{ Internal validation-corrected index $(95 \% \mathrm{Cl})^{*}$} \\
\cline { 3 - 5 } & & & Derivation cohort & Validation cohort \\
\hline AUC $(95 \% \mathrm{Cl})$ & $0.726(0.696-0.760)$ & $0.717(0.663-0.770)$ & $0.711(0.682,0.739)$ & $0.718(0.666,0.765)$ \\
Intercept & 0.000 & 0.000 & $0.021(-0.113,0.154)$ & $-0.012(-0.286,0.218)$ \\
Slope & 1.000 & 1.000 & $0.92(0.783,1.067)$ & $1.019(0.76,1.348)$ \\
Brier scaled & 0.207 & 0.207 & $0.213(0.203,0.224)$ & $0.209(0.194,0.229)$ \\
$R^{2}$ & 0.202 & 0.180 & $0.176(0.124,0.223)$ & $0.18(0.088,0.254)$ \\
\hline
\end{tabular}

${ }^{*}$, the 1,000 bootstrap resampling method was implied to perform internal validation in the derivation and validation cohort. AUC, area under receiver operating characteristic curve.

physiological functions include macronutrient metabolism, blood volume regulation, immune system support, endocrine control of growth signaling pathways, lipid and cholesterol homeostasis, and breakdown of exogenous compounds including many current drugs (13). Viral hepatitis, druginduced liver injury, hepatectomy, and other factors can cause temporary or permanent loss of liver function, and this pathophysiological state is called liver failure. Severe liver failure increases the patient's risk to secondary multiple organ failure and death. Currently, the residual liver volume of 25
$30 \%$ is generally used in clinical practice as the lower limit of necessary postoperative liver function in patients with normal liver function, while the minimum postoperative residual liver volume of patients with liver function impairment should not be less than $40 \%$ (14). However, it is difficult to accurately estimate the future residual liver volume before surgery using conventional imaging examination, and the risk factors associated with PHLF are not limited to the scope of hepatectomy. MLR is widely performed in hepatocellular carcinoma, hepatic hemangioma, cholelithiasis, and 
Table 4 Receiver operating characteristic analysis of prediction model for predicting the PHLF grade in total cohort

\begin{tabular}{lccc}
\hline Performance index & Grade $\geq A$ & Grade $\geq$ B & Grade $\geq$ C \\
\hline ROC & $0.723(0.697-0.750)$ & $0.792(0.735-0.850)$ & $0.821(0.720-0.922)$ \\
Cut-off & 172.63 & 188.57 & 209.02 \\
Specificity & $0.741(0.706-0.777)$ & $0.702(0.679-0.726)$ & $0.848(0.830-0.867)$ \\
Sensitivity & $0.604(0.572-0.638)$ & $0.778(0.667-0.873)$ & $0.714(0.524-0.905)$ \\
Accuracy & $0.661(0.637-0.686)$ & $0.705(0.683-0.728)$ & $0.847(0.829-0.865)$ \\
Negative predictive value & $0.573(0.550-0.597)$ & $0.986(0.979-0.992)$ & $0.995(0.992-0.998)$ \\
Positive predictive value & $0.765(0.738-0.792)$ & $0.107(0.092-0.122)$ & $0.066(0.047-0.083)$ \\
\hline
\end{tabular}

*, the optimal cut-off value of total points of prediction model were selected based on the Youden index.

other hepatobiliary diseases. Given the high volume of hepatectomy, the risk of PHH, PHLF, and even death is bound to increase, so the necessity, rationality, and related risks of MLR always affect the clinical decision-making of hepatobiliary surgery. Therefore, if the risk factors related to PHLF after MLR can be identified through preoperative routine imaging and laboratory examination and the risk of PHLF can be rapidly and accurately predicted, high-risk patients can be identified, which is essential for accurate planning of MLR.

The reported incidence of hepatectomy-related PHLF ranged from $0 \%$ to $43.1 \%$ (2). Thus far, many definitions and diagnosis systems are used for hepatectomy-related PHLF in clinical application, such as the "50-50 criteria" (15), peak total bilirubin criteria (16), and so on (6), which makes it difficult to conduct intuitive comparison and analysis of relevant studies with different standards. The unification of criteria and parameters for judging complications of hepatectomy is helpful to compare the results of different studies reliably, to evaluate the rationality of individualized hepatectomy schemes for different populations, and ultimately to provide reference for patients with the best interventional treatment. In 2010, the ISGLS defined PHLF as a postoperative deterioration in the ability of the liver to maintain its synthetic, excretory, and detoxifying functions, which are characterized by an increased international normalized ratio and concomitant hyperbilirubinemia on the operation day or days after the operation (6). Based on the analysis and study of relevant literature, ISGLS has formulated a concise and practical definition and grading of PHLF, and its rationality has been confirmed gradually by other studies (7). In this study, 1,441 MLR cases were analyzed using the ISGLS-PHLF definition and grading criteria, and the incidence of PHLF in this group was $58.22 \%$, implying that PHLF was not an adverse medical event with small probability in the patient population undergoing MLR. Overall, however, even if PHLF occurs following MLR, most of the patients have grade A PHLF (92.49\%) that is not life threatening and does not require a change in clinical treatment pathway, and the rate of severe grade C PHLF is only $1.46 \%$. Meanwhile, none of the other hepatectomy-related complications that required a change in clinical treatment pathway had an incidence of $>10 \%$ and a total perioperative mortality rate of $1.32 \%$.

Tzeng et al. found that surgical experience and volume, hospital-specific surgical safety culture, hepatectomy scope, and patient factors were the four main risk factors that were strongly associated with complications of hepatectomy (17). Compared with the results of other similar studies (i.e., perioperative mortality rate of $2.99-13.47 \%$ ) (2), our study demonstrates that the incidence of MLR-related adverse events can be restricted to a low level in high-volume hepatobiliary surgery centers, which is consistent with other research findings $(18,19)$.

Extensive hepatectomy has been found to be strongly associated with PHLF in a number of previous similar studies and has been incorporated into the PHLF prediction model (1,20-23). We have sought to achieve two main objectives in this study: (I) analyze sample data according to the ISGLS definition criteria, and we hope that more similar studies using the same research criteria will facilitate future controlled analysis of the results from different centers, (II) explore the establishment of a preoperative predictive model for liver failure after extensive hepatectomy, especially severe liver failure, using easily accessible preoperative clinical routine information on patients, and to validate the accuracy of the model to clarify the ease and 
reliability of the predictive model for clinical application. In the multivariate analyses, age, gender, preoperative serum platelet, creatinine, GGT, fibrinogen, HBe antigen positive, and number of resected liver segments were independent prognostic factors of PHLF for MLR patients, which were included ultimately in the nomogram. Some of these risk factors have been reported in other previous studies.

Several studies have found that male patients are more likely to develop PHLF $(21,24,25)$. Tzeng et al. (17) and Filicori et al. (24) both found a higher risk of serious complications after hepatectomy on older patients than on middle-aged patients. HBe antigen can accelerate liver injury by promoting the production of inflammatory cytokines (26). Liver tissue biopsies of patients who had been serum HBe antigen positive for more than 6 months revealed a state of persistent progression of liver tissue injury, even though the patients had a low serum viral load $(<2,000 \mathrm{IU} / \mathrm{mL})(27)$. Groeneveld et al. confirmed that the low level of plasma fibrinogen after MLR was associated with liver dysfunction and mortality in patients who underwent hepatectomy. In addition, they found that coagulation-dependent intrahepatic fibrin(ogen) deposition is a new mechanism to promote platelet aggregation and liver regeneration after partial hepatectomy (28).

Many studies reported about the correlation between preoperative serum creatinine level and PHLF (20,29-32). A study of PHLF after MLR reported that the change trend of creatinine and phosphate levels between the day of operation and the first day after operation can determine whether patients are at risk of PHLF and death (33). Similar to the above results, our statistical analysis revealed a significant correlation between preoperative serum creatinine concentration and PHLF, which was eventually incorporated into the prediction model of PHLF. However, we found that the preoperative increase in serum creatinine concentration in the univariate analysis was positively correlated with PHLF $(\mathrm{P}=0.027)$, while the two presented a negative correlation after multivariate analysis. The reason may be related to Simpson's paradox caused by the difference in the PHLF composition ratio between male and female patients our study (Table S4) (34).

Recently, platelets have shown critical importance during human liver regeneration $(35,36)$. There may be several mechanisms by which platelets promote liver regeneration after hepatectomy. A study found that the specific interaction between sinusoidal-endothelial cells and platelets was one of the key events that required sufficient regenerative response (37). Animal and human experiments have found that platelet-derived serotonin initiated liver regeneration after hepatectomy (38-40). Han et al. found that platelet counts and volume of platelet transfusion during liver transplantation were positively associated with early graft regeneration and that the association between platelets and post-transplantation graft regeneration was mediated by serotonin (41). The role of platelets in promoting liver regeneration after hepatectomy was also verified in a number of studies on the relationship between thrombocytopenia and liver dysfunction or failure $(9,23,42,43)$. In the present study, preoperative platelet levels were negatively correlated with the risk of PHLF after MLR, showing that the risk of PHLF was higher when preoperative platelet levels were lower.

GGT is a transferase, and its major function is to enable metabolism of glutathione and glutathionylated xenobiotics $(44,45)$. In the present study, multifactorial analysis showed that GGT was strongly associated with PHLF and found that the risk of PHLF was significantly elevated when patients had preoperative GGT more than three times the upper limit of the normal value in laboratory test. A recent study also found that elevated GGT was linked to an increased risk to a multitude of diseases and conditions, including cardiovascular disease, diabetes, metabolic syndrome, and all-cause mortality. The literature from multiple population groups worldwide consistently shows strong predictive power for GGT (46). The GGT-toplatelet ratio (GPR) is a noninvasive marker for assessing liver fibrosis. Liu et al. found that incorporating GPR into the model for end-stage liver disease may provide a more accurate survival prediction of 90 days in patients with acute-on-chronic liver failure (47). These findings strongly suggest that GGT may be a valid biomarker of liver reserve function and should be valued prior to hepatectomy.

As strengths of the present study, we recruited a large cohort to establish a stable predictive nomogram and internally validated. Although we know that AUCs are generally used to assess the accuracy of diagnostic models, if there are limitations in the multifactorial correlation analysis of risk factors associated with prediction models, then even if the results of the AUCs based on multifactor analysis are statistically significant, the accuracy and practical application of prediction models is still not assured. Misleading results are generally the main outcome of research that does not validate its prediction models. Therefore, to cover the potential risk factors for liver failure after hepatectomy as much as possible, we selected 48 analytic indicators for inclusion in this study based on 
literature reports and clinical practice. In addition, we crossvalidated both the derivation cohort and the validation cohort of the predictive model using 1,000 bootstraps. In this study, we also investigated the predicted AUCs and cut-off scores for different grades of PHLF in the whole population based on the ROC curve.

\section{Implications}

This study elucidates the current status of the major postoperative complications of MLR and their associated risk factors based on the results of a cohort analysis of a large sample. Although studies have shown that the risk of PHLF is significantly higher in liver resection range of $\geq 4$ liver segments than in liver resection range of $<4$ liver segments, the overall risk of serious adverse medical events after MLR resection that threaten patient recovery remains low. The results of the relevant studies contribute to the implementation of clinical decision-making for the rationalization of MLR.

\section{Acknowledgments}

We would like to thank Editage (www.editage.cn) for English language editing.

Funding: This study was supported by grants from the National Natural Science Foundation of China (81770613) and the Science and Technology Commission of Shanghai Municipality (19411967000).

\section{Footnote}

Reporting Checklist: The authors have completed the TRIPOD reporting checklist. Available at http://dx.doi. org/10.21037/atm-20-5472

Data Sharing Statement: Available at http://dx.doi. org/10.21037/atm-20-5472

Peer Review File: Available at http://dx.doi.org/10.21037/ atm-20-5472

Conflicts of Interest: All authors have completed the ICMJE uniform disclosure form (available at http://dx.doi. org/10.21037/atm-20-5472). All the authors report grants from National Natural Science Foundation of China and from Science and Technology Commission of Shanghai Municipality, during the conduct of the study.
Ethical Statement: The authors are accountable for all aspects of the work in ensuring that questions related to the accuracy or integrity of any part of the work are appropriately investigated and resolved. This study conformed to the provisions of the Declaration of Helsinki (as revised in 2013). This study has been approved by the Ethics Committee of Eastern Hepatobiliary Surgery Hospital (Ethics Audit No. EHBHKY2020-K-004). Each patient signed the clinical study informed consent form in person or by proxy.

Open Access Statement: This is an Open Access article distributed in accordance with the Creative Commons Attribution-NonCommercial-NoDerivs 4.0 International License (CC BY-NC-ND 4.0), which permits the noncommercial replication and distribution of the article with the strict proviso that no changes or edits are made and the original work is properly cited (including links to both the formal publication through the relevant DOI and the license). See: https://creativecommons.org/licenses/by-nc-nd/4.0/.

\section{References}

1. Lafaro K, Buettner S, Maqsood H, et al. Defining post hepatectomy liver insufficiency: where do we stand? J Gastrointest Surg 2015;19:2079-92.

2. Mehrabi A, Golriz M, Khajeh E, et al. Meta-analysis of the prognostic role of perioperative platelet count in posthepatectomy liver failure and mortality. Br J Surg 2018;105:1254-61.

3. van den Broek MA, van Dam RM, van Breukelen GJ, et al. Development of a composite endpoint for randomized controlled trials in liver surgery. Br J Surg 2011;98:1138-45.

4. Rahbari NN, Garden OJ, Padbury R, et al. Posthepatectomy haemorrhage: a definition and grading by the International Study Group of Liver Surgery (ISGLS). HPB (Oxford) 2011;13:528-35.

5. Koch M, Garden OJ, Padbury R, et al. Bile leakage after hepatobiliary and pancreatic surgery: a definition and grading of severity by the International Study Group of Liver Surgery. Surgery 2011;149:680-8.

6. Rahbari NN, Garden OJ, Padbury R, et al. Posthepatectomy liver failure: a definition and grading by the International Study Group of Liver Surgery (ISGLS). Surgery 2011;149:713-24.

7. Sultana A, Brooke-Smith M, Ullah S, et al. Prospective evaluation of the International Study Group for Liver Surgery definition of post hepatectomy liver failure after 
liver resection: an international multicentre study. $\mathrm{HPB}$ (Oxford) 2018;20:462-9.

8. Cescon M, Colecchia A, Cucchetti A, et al. Value of transient elastography measured with FibroScan in predicting the outcome of hepatic resection for hepatocellular carcinoma.Ann Surg 2012;256:706-12; discussion 712-3.

9. Prodeau M, Drumez E, Duhamel A, et al. An ordinal model to predict the risk of symptomatic liver failure in patients with cirrhosis undergoing hepatectomy. J Hepatol 2019;71:920-9.

10. Kim DK, Choi JI, Choi MH, et al. Prediction of posthepatectomy liver failure: MRI with hepatocytespecific contrast agent versus indocyanine green clearance test. AJR Am J Roentgenol 2018;211:580-7.

11. Couinaud C. Definition of hepatic anatomical regions and their value during hepatectomy (author's transl). Chirurgie 1980;106:103-8.

12. Steyerberg EW, Yvonne V. Towards better clinical prediction models: seven steps for development and an ABCD for validation. Eur Heart J 2014;35:1925-31.

13. Trefts E, Gannon M, Wasserman DH. The liver. Curr Biol 2017;27:R1147-R1151.

14. Vauthey JN, Abbott DE. Commentary on "Feasibility study of two-stage hepatectomy for bilobar liver metastases". Am J Surg 2012;203:698-9.

15. Balzan S, Belghiti J, Farges O, et al. The "50-50 criteria" on postoperative day 5: an accurate predictor of liver failure and death after hepatectomy. Ann Surg 2005;242:824-8.

16. Mullen JT, Ribero D, Reddy SK, et al. Hepatic insuffificiency and mortality in 1,059 noncirrhotic patients undergoing major hepatectomy. J Am Coll Surg 2007;204:854-62.

17. Tzeng CW, Cooper AB, Vauthey JN, et al. Predictors of morbidity and mortality after hepatectomy in elderly patients: analysis of 7621 NSQIP patients. HPB (Oxford) 2014;16:459-68.

18. Choti MA, Bowman HM, Pitt HA, et al. Should hepatic resections be performed at high-volume referral centers? J Gastrointest Surg 1998;2:11-20.

19. Dimick JB, Wainess RM, Cowan JA, et al. National trends in the use and outcomes of hepatic resection. J Am Coll Surg 2004;199:31-8.

20. Dasari BVM, Hodson J, Roberts KJ, et al. Developing and validating a pre-operative risk score to predict posthepatectomy liver failure. HPB (Oxford) 2019;21:539-46.

21. Liu JY, Ellis RJ, Hu QL, et al. Post hepatectomy liver failure risk calculator for preoperative and early postoperative period following major hepatectomy. Ann Surg Oncol 2020;27:2868-76.

22. Shirata C, Kokudo T, Arita J, et al. Albumin-indocyanine green evaluation (ALICE) grade combined with portal hypertension to predict post-hepatectomy liver failure. Hepatol Res 2019;49:942-9.

23. Golriz M, Ghamarnejad O, Khajeh E, et al. Preoperative thrombocytopenia may predict poor surgical outcome after extended hepatectomy. Can J Gastroenterol Hepatol 2018;2018:1275720.

24. Filicori F, Keutgen XM, Zanello M, et al. Prognostic criteria for postoperative mortality in 170 patients undergoing major right hepatectomy. Hepatobiliary Pancreat Dis Int 2012;11:507-12.

25. Bachellier P, Rosso E, Pessaux P, et al. Risk factors for liver failure and mortality after hepatectomy associated with portal vein resection. Ann Surg 2011;253:173-9.

26. Wang W, Bian H, Li F, et al. HBeAg induces the expression of macrophage miR-155 to accelerate liver injury via promoting production of inflammatory cytokines. Cell Mol Life Sci 2018;75:2627-41.

27. Oliveira VO, Oliveira JP, França EV, et al. Advanced liver injury in patients with chronic hepatitis B and viral load below 2,000 IU/mL. Rev Inst Med Trop Sao Paulo 2016;58:65.

28. Groeneveld D, Pereyra D, Veldhuis Z, et al. Intrahepatic fibrin(ogen) deposition drives liver regeneration after partial hepatectomy in mice and humans. Blood 2019; 133:1245-56.

29. Shen YN, Tang TY, Yao WY, et al. A nomogram for pred iction of posthepatectomy liver failure in patients with hepatocellular carcinoma: a retrospective study. Medicine (Baltimore) 2019;98:e18490.

30. Puri P, Lee WM, Fontana RJ, et al. Alcohol consumption is associated with the severity and outcome of acute liver injury/failure. Liver Int 2020;40:360-7.

31. Chen L, Zheng J, Cai J, et al. Predictive value of agebilirubin-international normalized ratio-creatinine score in short-term survival of acute-on-chronic hepatitis B liver failure. Cell Physiol Biochem 2018;51:2484-95.

32. Speiser JL, Karvellas CJ, Wolf BJ, et al. Predicting daily outcomes in acetaminophen-induced acute liver failure patients with machine learning techniques. Comput Methods Programs Biomed 2019;175:111-20.

33. Herbert GS, Prussing KB, Simpson AL, et al. Early trends in serum phosphate and creatinine levels are associated with mortality following major hepatectomy. HPB 
(Oxford) 2015;17:1058-65.

34. Rojanaworarit C. Misleading epidemiological and statistical evidence in the presence of Simpson's paradox: an illustrative study using simulated scenarios of observational study designs. J Med Life 2020;13:37-44.

35. Takahashi K, Liang C, Oda T, et al. Platelet and liver regeneration after liver surgery. Surg Today 2020;50:974-83.

36. Aryal B, Yamakuchi M, Shimizu T, et al. Therapeutic implication of platelets in liver regeneration -hopes and hues. Expert Rev Gastroenterol Hepatol 2018;12:1219-28.

37. Hagele S, Finsterbusch M, Fuxsteiner J, et al. Platelet to sinusoidal-endothelial-cell interaction during ongoing liver regeneration. Eur Surg 2018;50:S49.

38. Kirschbaum M, Jenne CN, Veldhuis ZJ, et al. Transient von Willebrand factor-mediated platelet influx stimulates liver regeneration after partial hepatectomy in mice. Liver Int 2017;37:1731-7.

39. Starlinger P, Pereyra D, Haegele S, et al. Perioperative von Willebrand factor dynamics are associated with liver regeneration and predict outcome after liver resection. Hepatology 2018;67:1516-30.

40. Yoshizumi T, Itoh S, Imai D, et al. Impact of platelets and serotonin on liver regeneration after living donor hepatectomy. Transplant Proc 2015;47:683-5.

41. Han S, Ko JS, Gwak MS, et al. Association of platelet

Cite this article as: Li B, Qin Y, Qiu Z, Ji J, Jiang X. A cohort study of hepatectomy-related complications and prediction model for postoperative liver failure after major liver resection in 1,441 patients without obstructive jaundice. Ann Transl Med 2021;9(4):305. doi: 10.21037/atm-20-5472 count and platelet transfusion with serotonin level during living donor liver transplantation: possible connection to graft regeneration. Transplant Proc 2018;50:1104-7.

42. Navarro JG, Yang SJ, Kang I, et al. What are the most important predictive factors for clinically relevant posthepatectomy liver failure after right hepatectomy for hepatocellular carcinoma? Ann Surg Treat Res 2020;98:62-71.

43. Meyer J, Balaphas A, Combescure C, et al. Systematic review and meta-analysis of thrombocytopenia as a predictor of post-hepatectomy liver failure. HPB (Oxford) 2019;21:1419-26.

44. Whitfield JB. Gamma glutamyl transferase. Crit Rev Clin Lab Sci 2001;38:263-355.

45. Wickham S, West MB, Cook PF, et al. Gammaglutamyl compounds: substrate specificity of gammaglutamyl transpeptidase enzymes. Anal Biochem 2011;414:208-14.

46. Koenig G, Seneff S. Gamma-Glutamyltransferase: a predictive biomarker of cellular antioxidant inadequacy and disease risk. Dis Markers 2015;2015:818570.

47. Liu L, Lan Q, Lin L, et al. Gamma-glutamyl transpeptidase-to-platelet ratio predicts the prognosis in $\mathrm{HBV}$-associated acute-on-chronic liver failure. Clin Chim Acta 2018;476:92-7. 


\section{Supplementary}

A

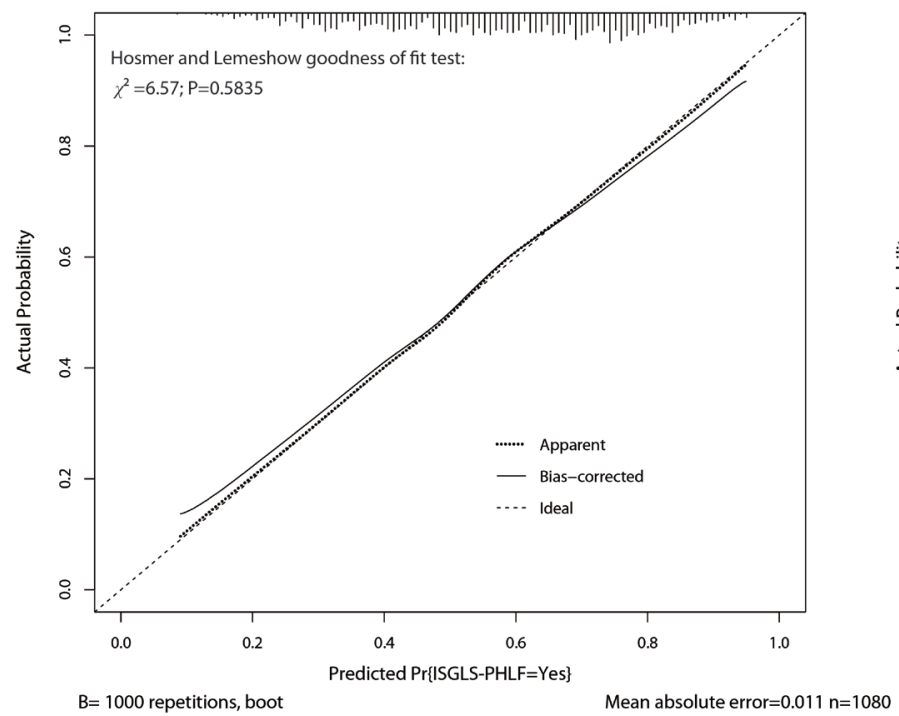

B

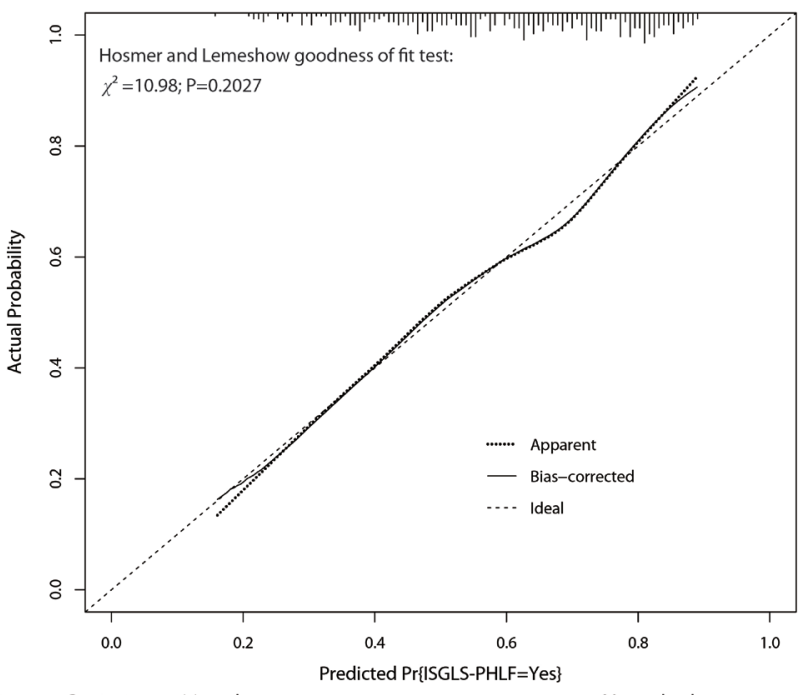

Mean absolute error $=0.012 \mathrm{n}=361$

Figure S1 The calibration plots for prediction models in derivation and validation cohort. (A) For derivation cohort; (B) for validation cohort.

A

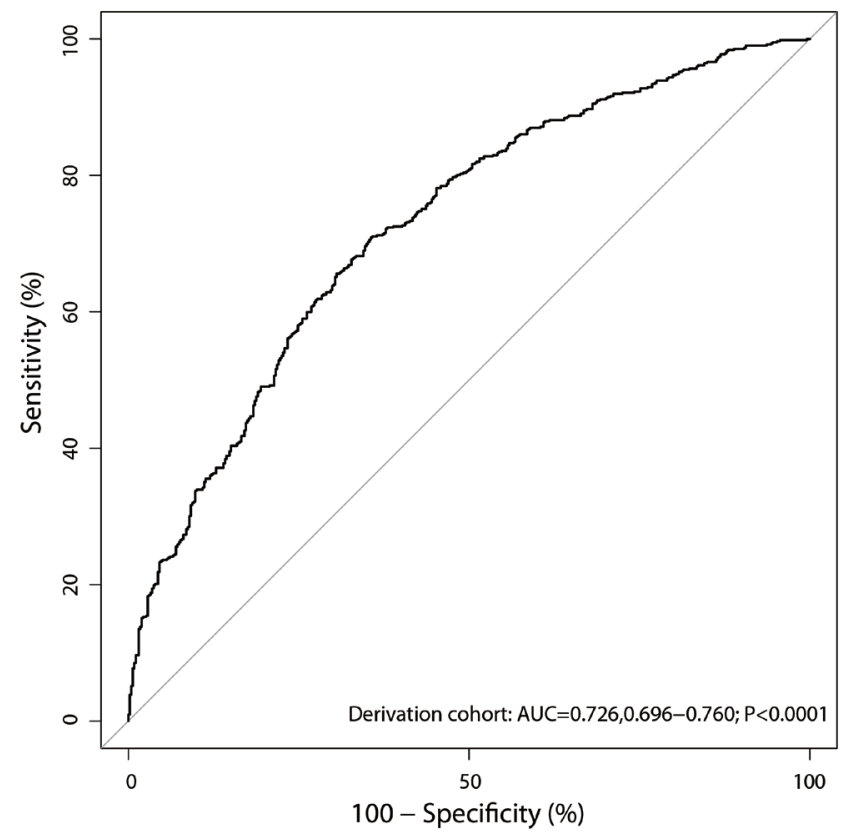

B



Figure S2 The receiver operating characteristic analysis of prediction models in derivation and validation cohort. 
A

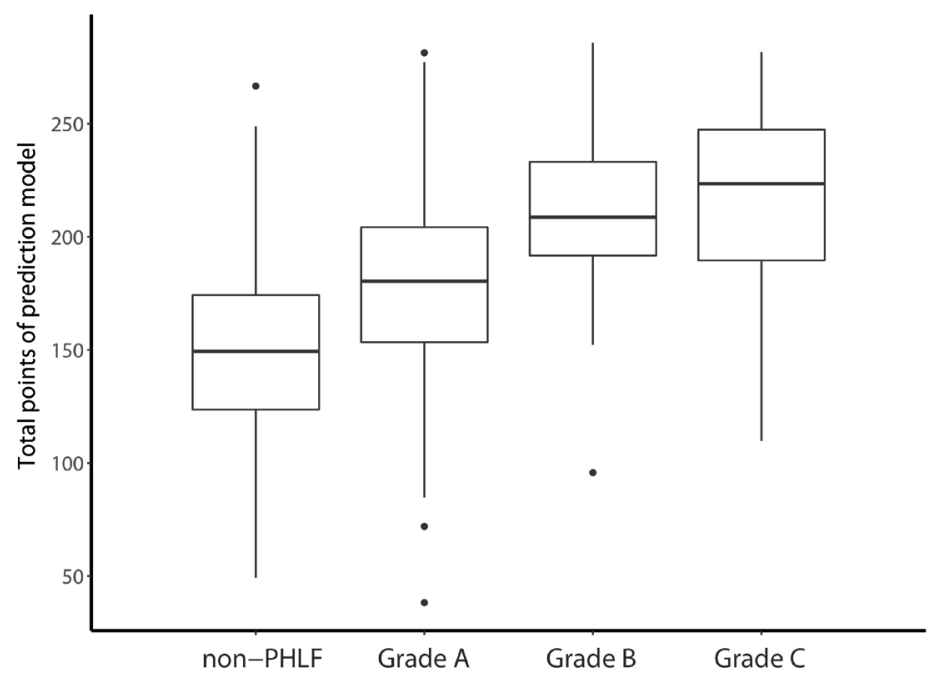

B

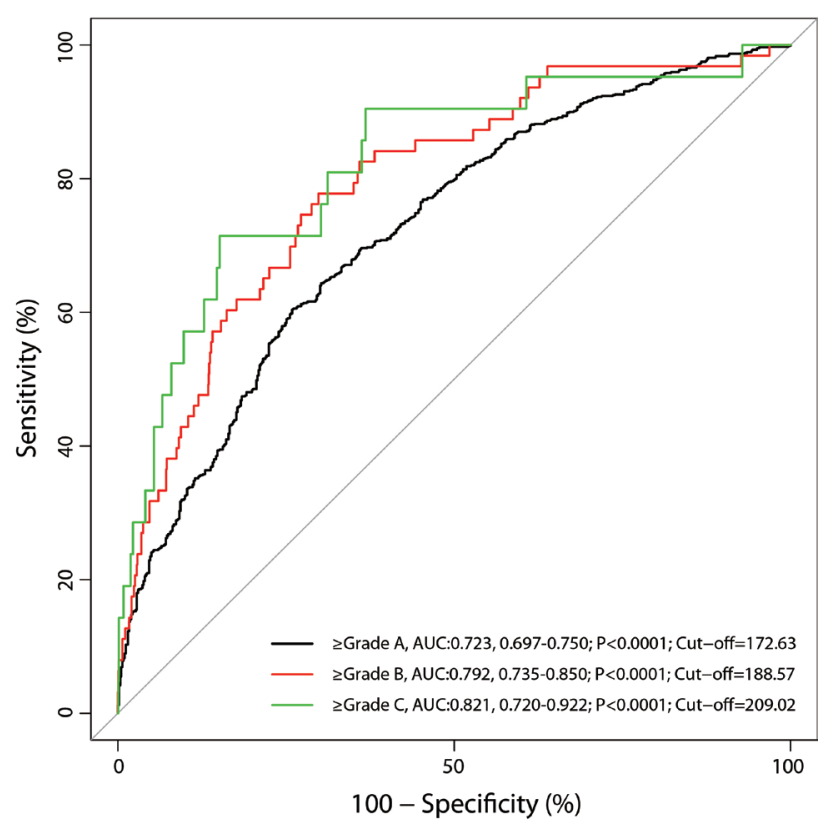

Figure S3 The box plot and receiver operating characteristic analysis for each ISGLS-PHLF grade in total cohort. (A) For box plot of total points in non-PHLF and three grades of PHLF, pairwise comparison with Nemenyi method indicated that all pairwise were significantly different except Grade B vs. Grade C ( $\mathrm{P}=0.9999)$; (B) for receiver operating characteristic analysis of three PHLF grades, and the cut-off value is obtained based on the Youden index. 
A



B

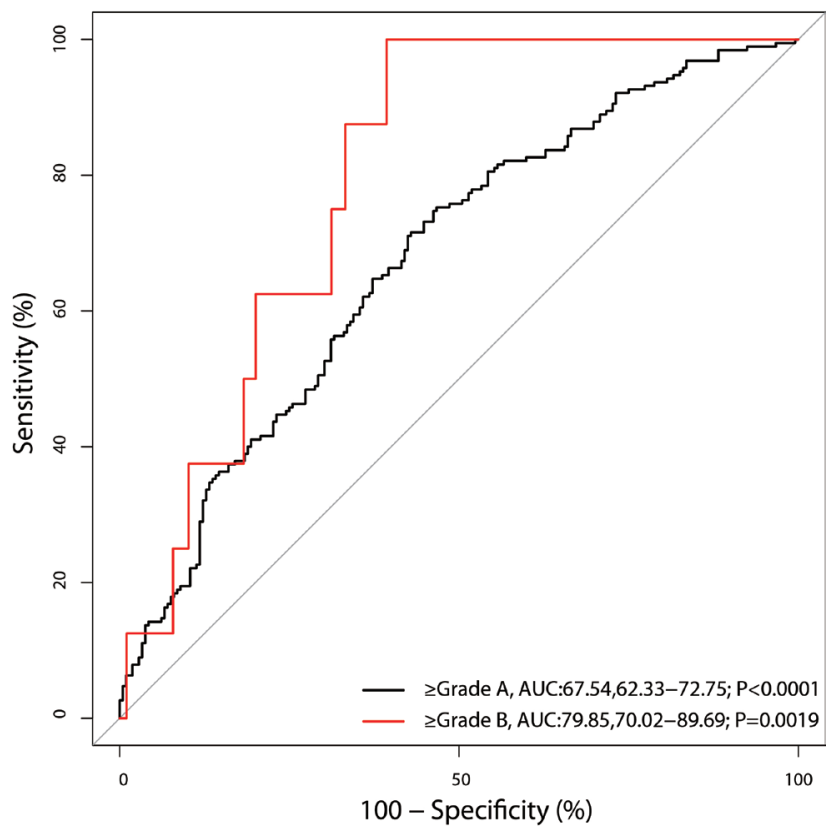

Figure S4 The receiver operating characteristic analysis for tumor and non-tumor disease in total cohort. (A) For common malignant neoplastic diseases of the liver, including hepatocellular carcinoma, intrahepatic cholangiocarcinoma, and metastatic hepatoma; (B) for hepatic hemangioma, intrahepatic cholelithiasis, and other rare occupational diseases of the hepatobiliary system (only 1 patient with grade C ISGLS-PHLF). 
Table S1 Demographics and baseline characteristics in the derivation and validation cohort

\begin{tabular}{|c|c|c|c|c|}
\hline Variable & Total cohort $(\mathrm{N}=1,441)$ & $\begin{array}{l}\text { Derivation cohort } \\
(\mathrm{N}=1,080)\end{array}$ & Validation cohort ( $\mathrm{N}=361)$ & Pvalue \\
\hline PHLF (yes) & $839(58.22)$ & $622(57.59)$ & $217(60.11)$ & 0.4010 \\
\hline ISGLS-PHLF & & & & 0.5463 \\
\hline Non-PHLF & $602(41.78)$ & $458(42.41)$ & $144(39.89)$ & \\
\hline Grade A & $776(53.85)$ & $579(53.61)$ & $197(54.57)$ & \\
\hline Grade B & $42(2.91)$ & $28(2.59)$ & $14(3.88)$ & \\
\hline Grade C & $21(1.46)$ & $15(1.39)$ & $6(1.66)$ & \\
\hline Gender (female) & $502(34.84)$ & $382(35.37)$ & $120(33.24)$ & 0.4623 \\
\hline Age & $52.00(44.00,60.00)$ & $52.00(45.00,60.00)$ & $52.00(43.00,60.00)$ & 0.7807 \\
\hline Blood type & & & & 0.9786 \\
\hline A & $477(33.10)$ & $347(32.13)$ & $130(36.01)$ & \\
\hline B & $371(25.75)$ & $290(26.85)$ & $81(22.44)$ & \\
\hline$A B$ & $144(9.99)$ & $115(10.65)$ & $29(8.03)$ & \\
\hline 0 & $449(31.16)$ & $328(30.37)$ & $121(33.52)$ & \\
\hline BMI & $22.68(20.69,24.90)$ & $22.66(20.62,24.91)$ & $22.86(20.76,24.68)$ & 0.8521 \\
\hline Diabetes & $23(1.60)$ & $14(1.30)$ & $9(2.49)$ & 0.1162 \\
\hline Cirrhosis & $264(18.32)$ & $202(18.70)$ & $62(17.17)$ & 0.5155 \\
\hline A long history of heavy drinking & $247(17.14)$ & $181(16.76)$ & $66(18.28)$ & 0.5061 \\
\hline History of chemotherapy before hepatectomy & & & & 0.3443 \\
\hline No & $1,300(90.22)$ & $980(90.74)$ & $320(88.64)$ & \\
\hline TAE & $124(8.61)$ & $87(8.06)$ & $37(10.25)$ & \\
\hline Systemic chemotherapy & $17(1.18)$ & $13(1.20)$ & $4(1.11)$ & \\
\hline Preoperative ascites & & & & 0.1980 \\
\hline No & $1,348(93.55)$ & $1,009(93.43)$ & 339 (93.91) & \\
\hline Mild & $92(6.38)$ & $71(6.57)$ & $21(5.82)$ & \\
\hline Moderate to severe & $1(0.07)$ & $0(0.00)$ & $1(0.28)$ & \\
\hline Preoperative serum hemoglobin level (<130 g/L) & $581(40.32)$ & $430(39.81)$ & $151(41.83)$ & 0.4996 \\
\hline Preoperative serum WBC count $\left(\times 10^{9} / \mathrm{L}\right)$ & $5.49(4.44,6.77)$ & $5.51(4.44,6.76)$ & $5.45(4.45,6.81)$ & 0.8512 \\
\hline Preoperative serum platelet count $\left(\times 10^{\circ} / \mathrm{L}\right)$ & $190.00(146.00,248.00)$ & $189.50(148.00,246.00)$ & $191.00(142.00,257.00)$ & 0.7326 \\
\hline Preoperative serum lymphocyte count $\left(\times 10^{\circ} \%\right.$ L) & $1.49(1.18,1.86)$ & $1.49(1.18,1.85)$ & $1.51(1.16,1.92)$ & 0.7288 \\
\hline Preoperative serum neutrophil count $\left(\times 10^{9} / L\right)$ & $3.35(2.58,4.49)$ & $3.36(2.59,4.49)$ & $3.33(2.55,4.49)$ & 0.9328 \\
\hline Preoperative serum creatinine level (umo/L) & $64.00(55.00,74.00)$ & $64.00(55.00,74.00)$ & $64.00(56.00,73.00)$ & 0.7251 \\
\hline $\mathrm{Na}+(\mathrm{mmol} / \mathrm{L})$ & $142.00(140.00,143.00)$ & $142.00(140.00,143.00)$ & $142.00(140.00,143.00)$ & 0.6233 \\
\hline $\mathrm{K}+(\mathrm{mmol} / \mathrm{L})$ & $4.11(3.90,4.34)$ & $4.11(3.92,4.34)$ & $4.13(3.90,4.34)$ & 0.5738 \\
\hline TBIL (umo/L) & $12.40(9.40,16.40)$ & $12.20(9.40,16.10)$ & $12.60(9.50,17.30)$ & 0.1083 \\
\hline DBIL (umo/L) & $4.70(3.50,6.30)$ & $4.70(3.50,6.30)$ & $5.00(3.60,6.40)$ & 0.2432 \\
\hline IBIL (umo/L) & $7.40(5.60,9.80)$ & $7.30(5.70,9.60)$ & $7.70(5.60,10.30)$ & 0.0935 \\
\hline TBA (umol/ml) & & & & 0.2419 \\
\hline$<2.7$ & $225(15.61)$ & $166(15.37)$ & $59(16.34)$ & \\
\hline $2.7-5.4$ & $405(28.11)$ & 316 (29.26) & $89(24.65)$ & \\
\hline$\geq 5.4$ & $811(56.28)$ & $598(55.37)$ & $213(59.00)$ & \\
\hline ALT ( $\geq 50 \mathrm{U} / \mathrm{L})$ & $316(21.93)$ & $230(21.30)$ & $86(23.82)$ & 0.3152 \\
\hline AST ( $(40 \mathrm{U} / \mathrm{L})$ & $534(37.06)$ & $389(36.02)$ & $145(40.17)$ & 0.1578 \\
\hline LDH ( $\geq 250$ U/L) & $217(15.06)$ & $164(15.19)$ & $53(14.68)$ & 0.8168 \\
\hline GGT (U/L) & & & & 0.5021 \\
\hline$<60$ & $458(31.78)$ & $351(32.50)$ & $107(29.64)$ & \\
\hline 60-180 & $600(41.64)$ & $441(40.83)$ & $159(44.04)$ & \\
\hline$\geq 180$ & $383(26.58)$ & $288(26.67)$ & 95 (26.32) & \\
\hline TP $(g / L)$ & $70.10(66.20,73.80)$ & $70.10(66.20,73.75)$ & $70.20(66.60,74.10)$ & 0.7159 \\
\hline ALB $(g / L)$ & $41.50(39.00,43.90)$ & $41.50(39.00,43.90)$ & $41.50(38.80,43.90)$ & 0.9914 \\
\hline GLB (g/L) & $28.20(25.40,31.50)$ & $28.10(25.40,31.50)$ & $28.50(25.50,31.50)$ & 0.5138 \\
\hline pALB (g/L) & $221.00(169.00,270.00)$ & $223.00(169.00,273.00)$ & $215.00(169.00,260.00)$ & 0.1097 \\
\hline APTT (s) & $27.10(24.90,30.10)$ & $27.20(24.90,30.10)$ & $27.10(25.10,30.30)$ & 0.7273 \\
\hline $\begin{array}{l}\text { Preoperative serum fibrinogen } \\
\text { concentration }(g / L)\end{array}$ & $2.82(2.29,3.57)$ & $2.80(2.26,3.58)$ & $2.88(2.30,3.53)$ & 0.5309 \\
\hline$\Pi(s)$ & $19.20(18.20,20.20)$ & $19.20(18.30,20.15)$ & $19.10(18.20,20.20)$ & 0.6158 \\
\hline PT (s) & $11.50(10.90,12.20)$ & $11.50(10.90,12.20)$ & $11.50(11.00,12.20)$ & 0.8054 \\
\hline INR & $0.96(0.91,1.02)$ & $0.96(0.91,1.02)$ & $0.97(0.92,1.02)$ & 0.7282 \\
\hline Child-Pugh grade & & & & 0.7412 \\
\hline Grade A & $1429(99.17)$ & $1072(99.26)$ & $357(98.89)$ & \\
\hline Grade B & $12(0.83)$ & $8(0.74)$ & $4(1.11)$ & \\
\hline HCV-Ab (positive) & $18(1.25)$ & $15(1.39)$ & $3(0.83)$ & 0.5806 \\
\hline HBs-Ag (positive) & 731 (50.73) & $550(50.93)$ & $181(50.14)$ & 0.7956 \\
\hline HBs-Ab (positive) & $454(31.51)$ & $338(31.30)$ & $116(32.13)$ & 0.7670 \\
\hline HBe-Ag (positive) & $167(11.59)$ & $123(11.39)$ & $44(12.19)$ & 0.6812 \\
\hline HBe-Ab (positive) & $822(57.04)$ & 626 (57.96) & 196 (54.29) & 0.2227 \\
\hline HBc-Ab (positive) & $1223(84.87)$ & $918(85.00)$ & 305 (84.49) & 0.8140 \\
\hline Liver segment count excised & & & & 0.5925 \\
\hline 3 & $742(51.49)$ & $549(50.83)$ & $193(53.46)$ & \\
\hline $3+$ & $120(8.33)$ & $95(8.80)$ & $25(6.93)$ & \\
\hline 4 & $423(29.35)$ & $318(29.44)$ & $105(29.09)$ & \\
\hline $4+$ & $112(7.77)$ & $82(7.59)$ & $30(8.31)$ & \\
\hline$\geq 5$ & $44(3.05)$ & $36(3.33)$ & $8(2.22)$ & \\
\hline $\begin{array}{l}\text { The segment of liver planned for resection has } \\
\text { atrophied (yes) }\end{array}$ & $320(22.21)$ & $248(22.96)$ & $72(19.94)$ & 0.2323 \\
\hline $\begin{array}{l}\text { The planned reserved segment of the liver has } \\
\text { developed compensatory hyperplasia (yes) }\end{array}$ & $77(5.34)$ & $53(4.91)$ & $24(6.65)$ & 0.2030 \\
\hline Pathological diagnostic classification & & & & 0.3707 \\
\hline Hepatocellular carcinoma & $728(50.52)$ & $535(49.54)$ & $193(53.46)$ & \\
\hline Intrahepatic cholangiocarcinoma & $279(19.36)$ & $204(18.89)$ & $75(20.78)$ & \\
\hline Hepatic hemangioma & $59(4.09)$ & $48(4.44)$ & $11(3.05)$ & \\
\hline Intrahepatic cholelithiasis & $270(18.74)$ & $213(19.72)$ & $57(15.79)$ & \\
\hline Metastatic hepatoma & $32(2.22)$ & $23(2.13)$ & $9(2.49)$ & \\
\hline $\begin{array}{l}\text { Other rare occupational diseases of the } \\
\text { hepatobiliary system }\end{array}$ & $73(5.07)$ & $57(5.28)$ & $16(4.43)$ & \\
\hline
\end{tabular}

Data are shown as $n(\%)$ or median (range). 
Intra-operative procedure
Intraoperative hepatic portal block

No

Intraoperative hepatic portal intermittent block

No

Total intraoperative hepatic portal block time (min)

Intraoperative blood loss (mL)

Intraoperative blood loss grading

Small

Moderate

Large

Intraoperative infusion of erythrocyte suspension

Yes

Total amount of intraoperative infusion of erythrocyte (unit)

Posthepatectomy outcome

sthepatectomy ascites

No

Yes

ISGLS-PHBL

No

Grade A

Grade

Grade C

No

Grade A
Grade B

Grade C

Posthepatectomy intra-abdominal abscess

No

Postoperative massive pleural effusion

No

$392(65.12) \quad 611(72.82)$

$\begin{array}{lll}528(87.71) & 700(83.43) & 0.0241\end{array}$

$\begin{array}{lll}15.00(0.00,21.00) & 18.00(0.00,25.00) & 0.0001\end{array}$

$300.00(200.00,500.00) \quad 400.00(200.00,800.00) \quad<0.000$

$504(83.72)<613(7306)-0.000$

$81(13.46) \quad 180(21.45)$

$17(2.82) \quad 46(5.48)$

$494(82.06)-572(68.18)-00.000$

$108(17.94)-267(31.82)$

$\begin{array}{lll}0.00(0.00,0.00) & 0.00(0.00,3.00) & <0.000\end{array}$

$<0.000$

$714(85.10$

$15(2.49) \quad 125(14.90)$

$554(92.03) \quad 726(86.53)$

$13(2.16) \quad 9(1.07)$

$34(5.65) \quad 103(12.28)$

$1(0.17) \quad 1(0.12)$

$573(95.18) \quad 713(84.98)$

$23(3.82) \quad 88(10.49)$

$\begin{array}{ll}6(1.00) & 28(3.34) \\ 0(0.00) & 10(1.19)\end{array}$

(20)

$590(98.01) \quad 791(94.28)$

$12(1.99) \quad 48(5.72)$

$580(96.35) \quad 781(93.09)$

$21(3.49) \quad 58(6.91)$

$1(0.17) \quad 0(0.00)$

Left

Perioperative death
№
$602(100.00)$
820 (97.74)
$0(0.00) \quad 19(2.26)$ Dakage; PHH, posthepatectomy haemorrhage. 


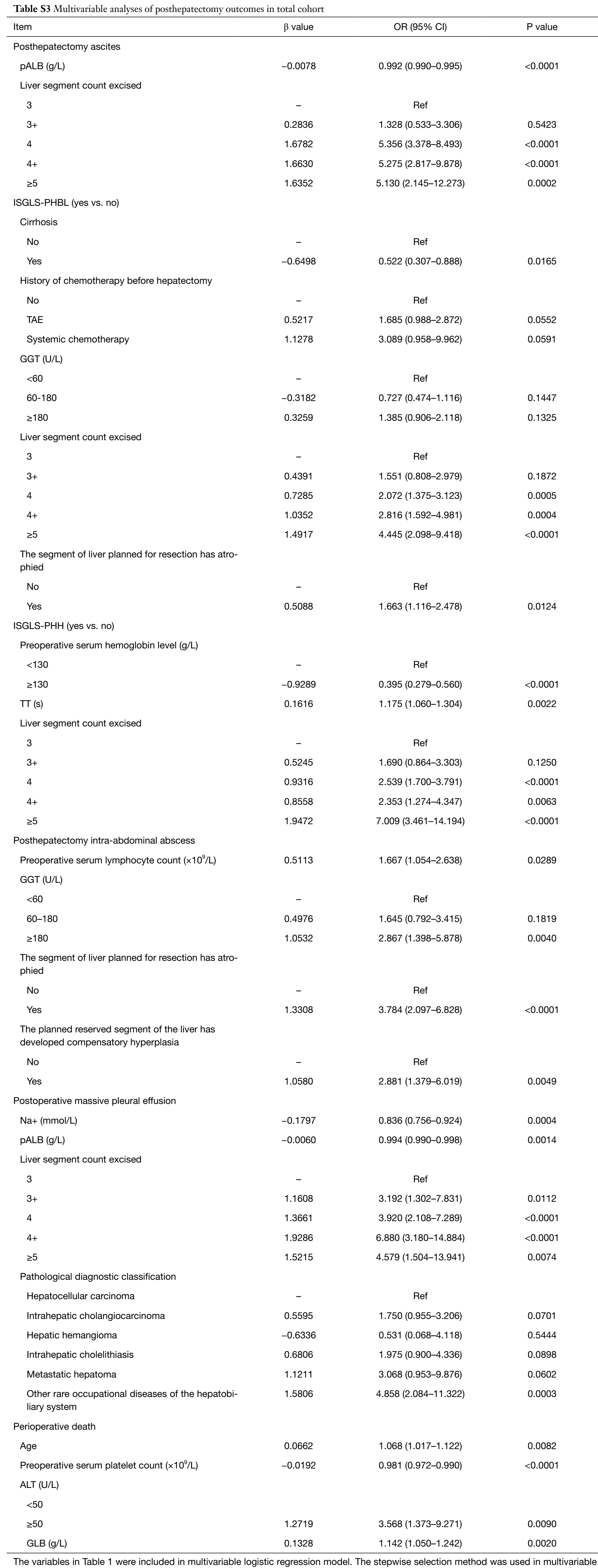

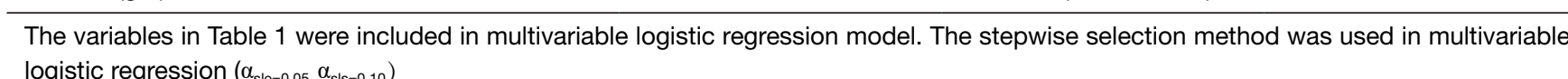


Table S4 Analyses of preoperative serum creatinine level in male and female patients (umol/L)

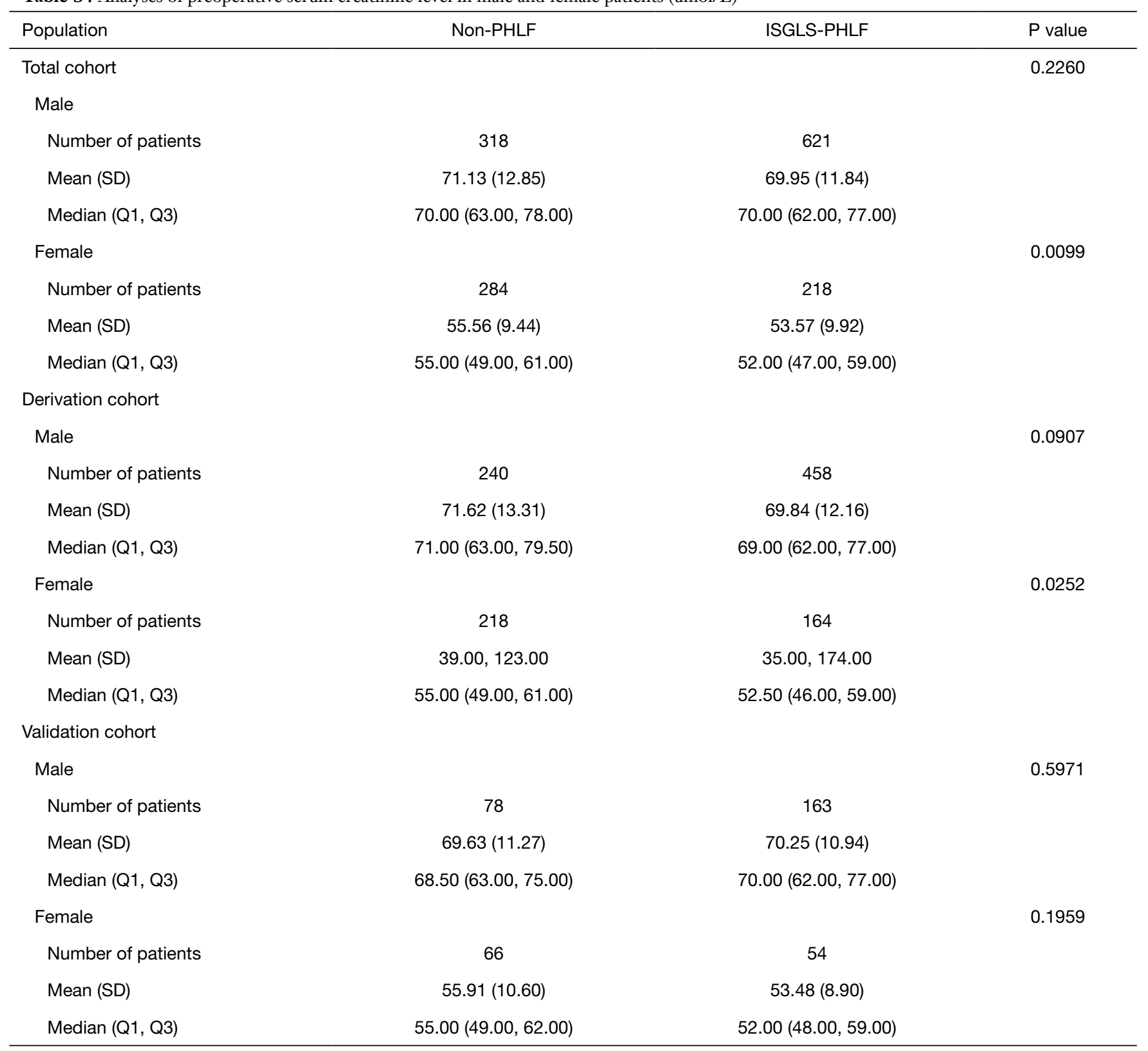

Data are shown as $\mathrm{n}(\%)$ or median (range). 